\title{
Surgical management of sternoclavicular septic arthritis
}

\author{
Thomas Nusselt $\cdot$ Hans-Michael Klinger $\cdot$ \\ Sven Freche $\cdot$ Wolfgang Schultz $\cdot$ Mike H. Baums
}

Received: 18 May 2010 / Published online: 20 August 2010

(C) The Author(s) 2010. This article is published with open access at Springerlink.com

\begin{abstract}
Introduction Septic arthritis of the sternoclavicular joint (SCJ) is a rare condition and has many diagnostic and therapeutic standards. The purpose of this study was to evaluate our experience with surgical and diagnostic management to provide a surgical pathway to help surgeons treat this disease.

Method We retrospectively reviewed five patients who were managed surgically between 1999 and 2007. All patients underwent structured diagnostic and treatment protocols. The functional outcome was evaluated using the Constant Score.

Patients The patients had the following underlying medical conditions: laryngeal cancer, port-explantation linked to a rectum carcinoma, spondylodiscitis, and brain stem infarct with reduced general condition; one patient had no underlying medical problems. Three patients underwent a simple incision, debridement and drainage, and two patients underwent an extended intervention with partial resection of the sternoclavicular joint. The mean duration of follow-up was 29 months (range 24-36 months). All patients had wellhealed wounds without signs of reinfection. The Constant Score for the functional outcome at the time of the last follow-up was 76 points (range 67-93 points). All patients recovered completely from SCJ disease.

Conclusion Our recommendations for the management of septic arthritis of the sternoclavicular joint include standard treatment steps and assessments. The early stages of infec-
\end{abstract}

T. Nusselt $(\bowtie) \cdot$ H.-M. Klinger $\cdot$ S. Freche $\cdot$ W. Schultz

M. H. Baums

Department of Orthopaedic Surgery,

University of Goettingen Medical Centre (UMG),

Georg-August-University, Robert-Koch-Str. 40,

37075 Göttingen, Germany

e-mail:dr.nusselt@gmx.net tion can be managed by simple incision, debridement and drainage. In advanced stages of infection, a more radical intervention is preferable.

Keywords Septic arthritis - Sternoclavicular joint . Surgical management · Treatment

\section{Introduction}

Septic arthritis of the sternoclavicular joint (SCJ) is a rare complication $[2,6,8,14,15,17]$ and is associated with a number of concomitant diseases. Diabetes mellitus, rheumatoid arthritis, intravenous drug use, intraarticular injection and immunosuppressive disorders are considered predisposing factors [3, 7, 10, 20, 23].

The SCJ is involved in only $0.5-1 \%$ of all infectious arthritis cases $[1,6,19]$. Nevertheless, a delay in the diagnosis of SCJ septic arthritis may lead to complications such as empyema [4], mediastinitis [16], osteomyelitis [21] or large abscess formations $[11,22]$. Additionally, Staphylococcus aureus, including methicillin-resistant $S$. aureus strains (MRSA), is becoming increasingly prevalent $[6,8$, $12,19]$.

Urgent surgical debridement should be supported by appropriate antibiotics; otherwise, there is a high risk of irreversible tissue damage and possible mortality [6, 8]. Joint and bone infections should be treated by intensive and specialised therapy [20].

Imaging technologies like computed tomography (CT) and magnetic resonance imaging (MRI) are used to determine the severity of an infection [2, 20].

The surgical management of septic arthritis of the SCJ has been recently discussed in the literature. There is controversy regarding the best method of joint decompression 
because good results have been reported using different treatment approaches ranging from a simple incision to en bloc resection with muscle flaps $[2,3,8,20]$.

The purpose of this study was to present our experience with the surgical management of septic arthritis of the SCJ. Furthermore, a flowchart was developed to help surgeons treat this condition.

\section{Patients and methods}

We retrospectively reviewed patients who were admitted to our department with septic arthritis of the SCJ between 1999 and 2007. Five patients with a mean age of 60 years (range 31-83 years) at the time of surgery were identified. A review of each patient's medical records, including associated predisposing factors and comorbidities, laboratory results, radiographic imaging and treatment modalities, was performed.

Patients were evaluated by laboratory studies including a complete blood cell count and measurements of C-reactive protein (CRP) levels and erythrocyte sedimentation rates (ESRs). Standard radiographs and CT or MR imaging were also performed on all patients. Aspiration of the affected sternoclavicular joint was performed preoperatively in all patients. Septic arthritis of the SCJ was confirmed by a positive culture from the joint fluid or by surgical specimens. No antibiotics were given before surgery to avoid influencing the identification of any causative organisms. The most recent follow-up that included a clinical evaluation was used as the basis for shoulder function. The functional outcome was evaluated using the Constant Score [5].

All patients were definitively diagnosed with septic arthritis and thus underwent immediate surgery depending on the extent of infection.

\section{Surgical techniques and postoperative management}

Two operative techniques were used in this series as determined by the severity of CT/MRI findings. Open-joint exploration with incision, debridement and drainage was performed in patients under general anaesthesia when the CT/MRI scan revealed abscesses around the SCJ without bony involvement. Patients were positioned in beach chair position. A hockey-stick type incision that starts from the medial third of the clavicle on the affected side to the manubrium sterni and then moves down to the sternomanubrial junction was used $[2,20]$. Any severely infected skin overlying the SCJ was included with the specimen. The entire anterior entire aspect of the joint capsule was then opened. Any necrotic and infected soft tissue was debrided [3], and the joint was appropriately irrigated with saline solution. Drains were placed and the wounds were closed.
When CT/MRI imaging demonstrated osseous destruction or radiological signs of osteomyelitis, a partial resection of the SCJ, including the surrounding infected soft tissue, was performed. A hockey-stick type incision was also used in these cases. The muscular attachments from the clavicle and the manubrium (M. sternocleidomastoideus and M. pectoralis major) were divided, and the underlying soft tissue was separated from the bony structures. Careful attention was given to the underlying vascular structures during these procedures. The discus of the sternoclavicular joint was then resected, and the cartilaginous coating of the sternoclavicular joint was completely removed. Upon the debridement of the soft tissue, the wounds were irrigated with saline solution and closed after drain placement. Suction drainage catheters were placed beneath the muscle and left for $48 \mathrm{~h}$.

Postoperatively, intravenous antibiotics were administered for 14 days and continued orally for 6 weeks. Physical therapy, including active and passive range of motion exercises, was started on postoperative day 2 and continued after discharge.

\section{Results}

Our records included five patients with SCJ septic arthritis who underwent surgical treatment in our department. A synopsis of these cases is given in Table 1 .

All five patients were male; the patients had right-sided SCJ disease in four cases and left-sided SCJ disease in one case.

The mean duration of symptoms before presentation was 8 days (range 5-14 days). All patients complained of pain that radiated to their shoulder and tenderness and chest pain localised around the affected SCJ. Redness and swelling were only noted in three of the five patients. When they were first seen for infection, all five patients had fever with a temperature ranging from 37.8 to $39.3^{\circ} \mathrm{C}$, and all had a feeling of general malaise. On physical examination, four of five patients had a reduced range of motion of the involved shoulder. A laboratory analysis revealed an increase in inflammatory markers, but only two of the five patients had leukocytosis.

The patients had different underlying medical disease as follows: laryngeal cancer, rectal carcinoma-related portexplantation 10 months prior to presentation, spondylodiscitis 4 months prior to presentation, and brain stem infarct with reduced general condition. One patient was completely healthy with no history of trauma or joint irritation.

Every patient had initial plain radiographs that showed no hint of septic destruction. A CT scan was done in three cases (No. 3, No. 4 and No. 5) and MRI was done in two cases (No. 1 and No. 2). In these investigation tools signs of 


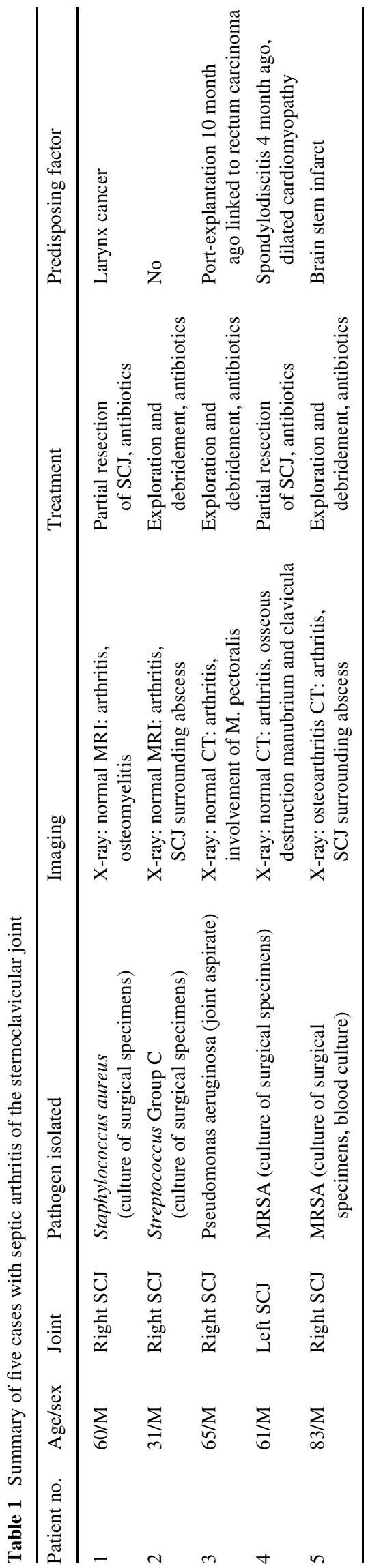

septic arthritis were found in all patients. In two cases (No. 2 and No. 5), arthritis and a sternoclavicular-surrounding abscess were found. One patient (No. 3) showed an involvement of the M. pectoralis, and osseous destruction of the medial clavicula and manubrium was observed in another patient (No. 4). Radiological signs of osteomyelitis were found in one case (No. 1).

Three of the five patients (No. 2, No. 3 and No. 5) underwent a simple incision, debridement and drainage procedure, while the other two (No. 1 and No. 4) underwent an extended intervention with partial resection of the sternoclavicular joint.

A causative organism was found in all cases. Pathogens were discovered by culture of the surgical specimens in four cases (one of which also had a positive blood culture) and from the joint aspirate in one case (No. 3).The following infectious organisms were identified: $S$. aureus in three cases, MRSA in two cases, Pseudomonas aeruginosa in one case, and Streptococcus Group C in one case.

The mean duration of follow-up was 29 months (range 24-36 months). All patients had well-healed wounds without signs of reinfection or any limitation to range of motion. The Constant Score [5] for the functional outcome at the time of the last follow-up was 76 points (range 67-93 points). All patients recovered completely from their SCJ infections.

\section{Discussion}

The principle results of our study show that therapy should always be based on a standard treatment protocol that includes surgery and appropriate antibiotics.

Infectious arthritis of the SCJ accounts for only $0.5-1 \%$ of all infectious arthritis cases [1, 6, 19]. Because of its rarity, an optimal therapy of SCJ septic arthritis has not yet been defined [20].

The surgical management of SCJ septic arthritis is a challenging problem. The joint's anatomical location near major vessels and its thin skin covering complicates surgical intervention [20]. The principles of management are eradication of infection, restoration of joint function and relief of pain. The high frequency of serious complications, such as empyema [4], mediastinitis [16], osteomyelitis [21] or large abscess formations $[11,22]$, increases the risk of irreversible tissue damage and possible mortality $[6,8]$.

There are a number of concomitant diseases associated with septic arthritis of the SCJ. Diabetes mellitus, rheumatoid arthritis, intravenous drug use, intraarticular injection and immunosuppressive disorders are predisposing factors $[3,7,10,15,23]$. There are several case reports of septic arthritis of the SCJ in healthy adults, including the patient in our study $[1,9,23]$. Therefore, septic arthritis should 
always be considered in the differential diagnosis of patients who present with painful, unilateral swelling of the SCJ or the surrounding region, especially with an acute onset [18].

Ross et al. [19] reported 180 cases of septic arthritis of the sternoclavicular joint; in accordance with our findings, $85 \%$ showed normal plain radiographs and at least one abnormality on CT or MRI, both of which are used to determine the severity of an infection (Fig. 1) [2, 13, 20].

Staphylococcus aureus was responsible for infection of the SCJ in three of our five cases, while MRSA was identified in two cases. Ross et al. [19] reported that the incidence of S. aureus was $49 \%$. S. aureus, including MRSA, is currently becoming increasingly prevalent and is the most commonly isolated infectious organism in septic SCJ $[6,8$, $12,19,23]$.

Carlos et al. [3] described eight patients with septic arthritis of the SCJ. An open-joint exploration with drainage and debridement was performed in four patients, while the remaining four patients had CT evidence of diffuse joint and surrounding bone destruction with involvement of the mediastinal soft tissue. The surgical therapy for these joint infections involved en bloc resection of the sternoclavicular joint and the ipsilateral pectoralis major muscle flap. There was complete healing with no apparent limitations in the range of motion in all patients, even after en bloc resection.

Song et al. [20] reported that six patients with a septic arthritis of the SCJ were treated by simple incision, drainage and debridement and were supported by antibiotic therapy. CT scans showed manubrial and clavicular erosion and the involvement of the surrounding tissue. They concluded that this procedure was ineffective and led to recurrence infection in five of the six patients. Therefore, these patients subsequently underwent a resection of their sternoclavicular joint. There were no wound complications and regular function of the upper limb was recovered at follow-up.

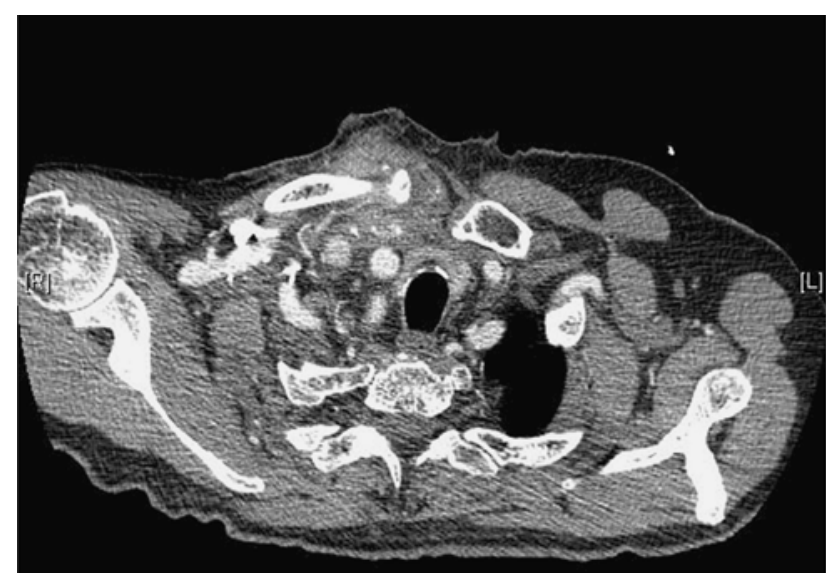

Fig. $1 \mathrm{CT}$ at the level of the right sternoclavicular joint demonstrating oedema and swelling of surrounding tissue
Burkhart et al. [2] performed a resection of the SCJ in 20 patients and a simple incision, debridement and drainage in 6 patients with septic arthritis of the SCJ. At least one abnormality (swelling, bone destruction or abscess) was found on CT scans. One patient died during hospitalisation as a result of sepsis. There were no criteria mentioned to indicate when incision and drainage versus resection is appropriate. Currently, the patients who survived do not show any signs of infection or limitations to range of motion.

There were five patients in our series: one with osseous destruction (No. 4), one with osteomyelitis (No. 1), and three with abscesses surrounding the SCJ (No. 2, No. 3 and No. 5). Our surgical management is closely comparable to Carlos et al. [3]; however, we employed a less aggressive approach. We dispensed with using a muscle flap, and this alteration may have caused a lower severity of infections. We also had no recurrence of infection, a complication that was described by Song et al. [20]. Additionally, Fordham et al. [8] presented one case in which they did a minimally invasive approach with washout of the sternoclavicular joint, despite CT scans that revealed osteomyelitis in the medial clavicle and manubrium. This patient also recovered completely.

Statements about potential long-term changes, such as for example, SCJ dislocation are not possible, because of our short-term follow-up with a mean of 29 months. Carlos et al. [3], Song et al. [20] and Burkhart et al. [2] also present follow-up between 20 and 28 months.

The disagreement about surgical management and the inconsistent guidelines for operative procedures are unsatisfying. The principles of correct management of SCJ septic arthritis should always be based on a structured diagnostic and treatment protocol. The developed flowchart (Fig. 2) should help surgeons in the systematic treatment of this rare condition. An early diagnosis is imperative for optimal treatment and helps to avoid prolonged hospitalisation and sequelae of chronic infection [3]. Surgical debridement and organism-specific antibiotics are integral to the eradication of infection of the SCJ. Surgical management depends on clinical and radiological findings [8]. If there is an early stage of infection, such as abscesses surrounding the SCJ without involvement of local structures, then simple incision and drainage may be successful. If there is an advanced stage of infection such as osteomyelitis, mediastinitis or bony destruction, an extended intervention is indicated [20].

\section{Conclusion}

Our recommendations for the management of septic arthritis of the SCJ include standard treatment steps and 


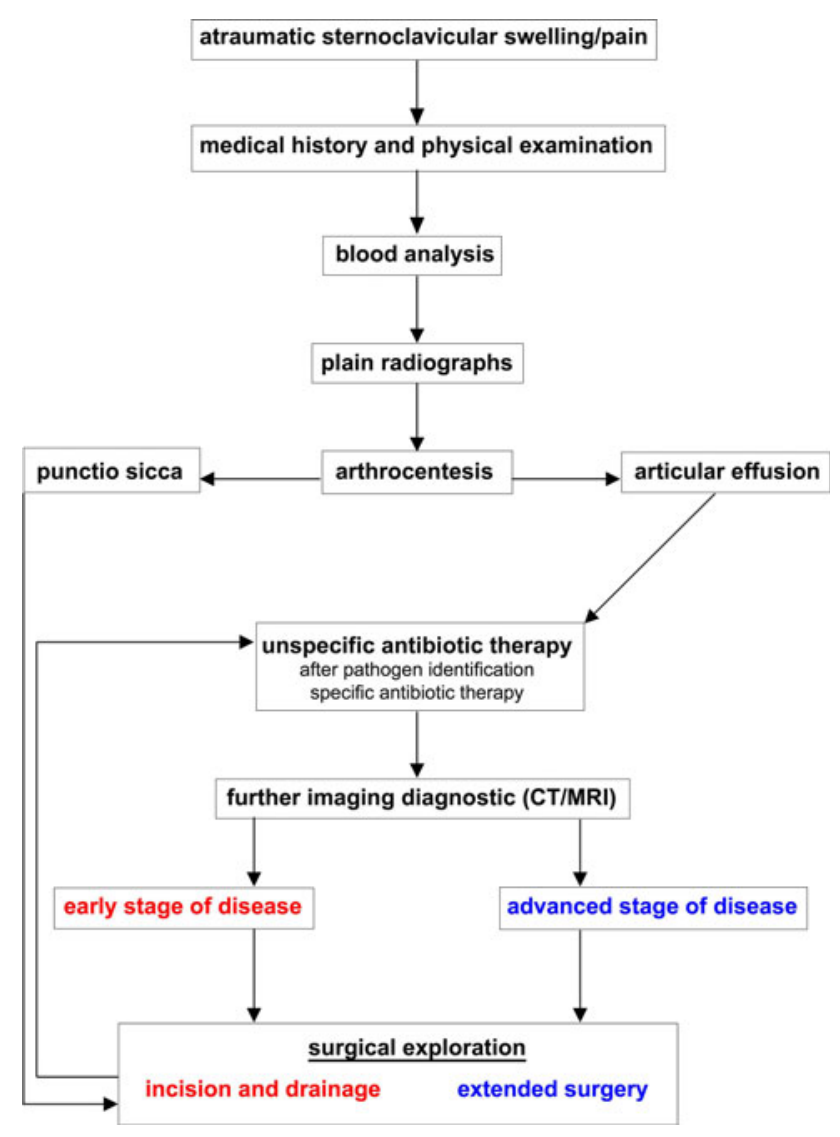

Fig. 2 Flowchart for diagnosis and medical treatment of septic arthritis of the sternoclavicular joint

assessments. For sufficient infection control, surgical debridement of infected tissue combined with suitable antibiotic therapy is essential. Our proposed diagnostic and clinical pathway describes the optimal management of septic arthritis of the SCJ. CT and MRI scans are indispensable for choosing the surgical strategy. The early stages of infection can be managed by simple incision, debridement and drainage. In advanced stages of infection, a more radical intervention is preferable.

Conflict of interest The authors declare that they have no conflicts of interest.

Open Access This article is distributed under the terms of the Creative Commons Attribution Noncommercial License which permits any noncommercial use, distribution, and reproduction in any medium, provided the original author(s) and source are credited.

\section{References}

1. Bar-Natan M, Salai M, Sisi Y, Gur H (2002) Sternoclavicular infectious arthritis in previously healthy adults. Semin Arthritis Rheum 32:189-195
2. Burkhart HM, Deschamps C, Allen MS, Nichols FC 3rd, Miller DL, Pairolero PC (2003) Surgical management of sternoclavicular joint infections. J Thorac Cardiovasc Surg 125:945-949

3. Carlos GN, Kesler KA, Colemann JJ, Broderick L, Turrentine MW, Brown JW (1997) Aggressive surgical management of sternoclavicular joint infections. J Thorac Cardiovasc Surg 113:242-247

4. Chen WS, Wan YL, Lui CC, Lee TY, Wang KC (1993) Extrapleural abscess secondary to infection of the sternoclavicular joint. J Bone Jt Surg Am 75:1835-1839

5. Constant CR, Murley AH (1987). A clinical method of functional assessment of the shoulder. Clin Orthop Rel Res (214):160-164

6. Crisostomo RA, Laskowski ER, Bond JR, Agerter DC (2008) Septic sternoclavicular joint: a case report. Arch Phys Med Rehabil 89:884-886

7. El Ibrahim A, Daoudi A, Boujraf S, Elmrini A, Boutayeb F (2009) Sternoclavicular septic arthritis in a previously healthy patient: a case report and a review of the literature. Int J Infect Dis 13:119-121

8. Fordham S, Cope S, Sach M (2009) Optimal management of sternoclavicular septic arthritis. Eur J Emerg Med 16:219-220

9. Gillis S, Friedman B, Caraco Y, Blankstein A, Yellin A, Friedman G (1990) Septic arthritis of the sternoclavicular joint in healthy adults. J Inter Med 228:275-278

10. Higginbotham TO, Kuhn JE (2005) Atraumatic disorders of the sternoclavicular joint. J Am Acad Orthop Surg 13:138-145

11. Lindhoudt DV, Velan F, Ott H (1989) Abscess formation in sternoclavicular septic arthritis. J Rheumatol 16:413-414

12. Lowy FD (1998) Medical progress: Staphylococcus aureus infections. N Engl J Med 339:520-532

13. Majeed A, Aschenbach R, Vorwerk D (2009) Acute ascending mediastinitis due to septic arthritis of the sternoclavicular joint. Rofo 181:1007-1008

14. Mikroulis DA, Verettas DA, Xarchas KC, Lawal LA, Kazakos KJ, Bougioukas GJ (2008) Sternoclavicular joint septic arthritis and mediastinitis. A case report and review of the literature. Arch Orthop Trauma Surg 128:185-187

15. Mozen PH, Zell SC (1988) Sternoclavicular bacterial arthritis. West J Med 148:310-312

16. Pollack MS (1990) Staphylococcal mediastinitis due to sternoclavicular pyarthrosis: CT appearance. J Comput Assist Tomogr 14:924-927

17. Prathap KK, Simpson D, Hunter SG (2000) Primary pyogenic arthritis of sterno-clavicular joint. Injury 31:267-268

18. Robinson CM, Jenkins PJ, Markham PE, Beggs I (2008) Disorders of the sternoclavicular joint. J Bone Jt Surg Br 90:685-696

19. Ross JJ, Shamsuddin H (2004) Sternoclavicular septic arthritis: review of 180 cases. Medicine 83:139-148

20. Song HK, Guy TS, Kaiser LR, Shrager JB (2002) Current presentation and optimal surgical management of sternoclavicular joint infection. Ann Thorac Surg 73:427-431

21. Tecce PM, Fishman EK (1995) Spiral CT with multiplanar reconstruction in the diagnosis of sternoclavicular osteomyelitis. Skelet Radiol 24:275-281

22. Wolgethan JR, Newberg AH, Reed JI (1995) The risk of abscess from sternoclavicular joint. A report of three cases. J Bone Jt Surg Am 77:136-139

23. Zanelli G, Sansoni A, Migliorini L, Donati E, Cellesi C (2003) Sternoclavicular joint infection in an adult without predisposing risk factors. Infez Med 1:105-107 